\title{
Your Goal Is Mine: Unraveling Mimetic Desires in the Human Brain
}

\author{
Maël Lebreton, ${ }^{1,2,3}$ Shadia Kawa, ${ }^{1,3}$ Baudouin Forgeot d'Arc, ${ }^{4,5}$ Jean Daunizeau, ${ }^{1,3}$ and Mathias Pessiglione ${ }^{1,2,3}$ \\ ${ }^{1}$ Motivation, Brain and Behavior Team and ${ }^{2}$ Centre de NeuroImagerie de Recherche, Institut du Cerveau et de la Moelle épinière, Hôpital Pitié-Salpêtrière, \\ 75013, Paris, France, ${ }^{3}$ Institut National de la Santé et de la Recherche Médicale, Centre National de la Recherche Scientifique, Université Pierre et Marie \\ Curie, 75005 Paris, France, ${ }^{4}$ Laboratoire de Sciences Cognitives et Psycholinguistique, Ecole Normale Supérieure, 75005 Paris, France, and ${ }^{5}$ Service de \\ Psychiatrie de l'Enfant et de l'Adolescent, Hôpital Robert Debré, 75019 Paris, France
}

The spread of desires among individuals is widely believed to shape motivational drives in human populations. However, objective evidence for this phenomenon and insights into the underlying brain mechanisms are still lacking. Here we show that participants rated objects as more desirable once perceived as the goals of another agent's action. We then unravel the mechanisms underpinning such goal contagion, using functional neuroimaging. As expected, observing goal-directed actions activated a parietofrontal network known as the mirror neuron system (MNS), whereas subjective desirability ratings were represented in a ventral striatoprefrontal network known as the brain valuation system (BVS). Crucially, the induction of mimetic desires through action observation involved the modulation of BVS activity through MNS activity. Furthermore, MNS-BVS effective connectivity predicted individual susceptibility toward mimetic desires. We therefore suggest that MNS-BVS interaction represents a fundamental mechanism explaining how nonverbal behavior propagates desires without the need for explicit, intentional communication.

\section{Introduction}

In a playground, children often run after the same toy, even if identical toys are present nearby. This is a canonical example of the "mimetic desire" phenomenon, a concept coined by the philosopher René Girard (Girard, 1988). The basic idea is that desires not only depend on the intrinsic properties of objects but also on whether these objects seem as an attractive goal to others. In social psychology, a goal is defined as a mental representation of a desired state (Gollwitzer and Moskowitz, 1996). Within this field, the mimetic desire phenomenon is recognized as a case of "goal contagion": objects that appear to represent a goal for another person tend to become a goal for the observer. In previous experiments designed by social psychologists, goals were transmitted to the subject merely by written information about others' behavior (Aarts et al., 2004), which leaves open the possibility of deliberate, strategic inference, as opposed to implicit, automatic contagion. Here we set up a series of experiments demonstrating this phenomenon in a basic nonverbal situation in which participants watched an actor performing an action directed toward an object.

\footnotetext{
Received Sept. 21, 2011; revised Feb. 24, 2012; accepted March 15, 2012.

Author contributions: S.K., B.F.d.A., and M.P. designed research; M.L. and S.K. performed research; J.D. contributed unpublished reagents/analytic tools; M.L. and M.P. analyzed data; M.L. and M.P. wrote the paper.

The work was supported by the Paris School of Neuroscience. M.L. was supported by a Fellowship from the French Ministère de l'Education Nationale. We thank Julie Grèzes, Philippe Fossati, and Chris Frith for their valuable comments on the manuscript. The English was edited by Beth Pavlicek and Alina Strasser.

Correspondence should be addressed to Mathias Pessiglione, Motivation, Brain and Behavior Team, Institut du Cerveau et de la Moelle épinière, 75013 Paris, France. E-mail: mathias.pessiglione@gmail.com.

DOI:10.1523/JNEUROSCI.4821-11.2012

Copyright $\odot 2012$ the authors $\quad 0270-6474 / 12 / 327146-12 \$ 15.00 / 0$
}

We also addressed the question of the neural mechanisms underlying goal contagion. Previous functional neuroimaging studies (Iacoboni et al., 1999; Grezes et al., 2003; Johnson-Frey et al., 2003) have shown that observing an action yields neuronal activation in a brain network including parietal lobules (PLs) and premotor cortex (PMC). These regions were labeled as components of a mirror neuron system (MNS) because they are activated both when performing an action and when observing an action being performed by someone else (Iacoboni and Dapretto, 2006; Van Overwalle and Baetens, 2009; Rizzolatti and Sinigaglia, 2010). We suggest that, beyond automatic elicitation of action representations, MNS activation may affect the observer's own motivational system, increasing the desirability of objects pursued by others.

In economic decision theories, desirability is often referred to as a "goal value." When facing a choice, the economic agent is supposed to valuate the available options and then select the one with the highest expected value (Von Neumann and Morgenstern, 1944; Rangel et al., 2008). Functional MRI (fMRI) studies have identified the neural correlates of stimuli values not only in choice situations but also when subjects watch passively, give pleasantness ratings, or perform a distractive task (Hare et al., 2009; Lebreton et al., 2009; Levy et al., 2011). Brain regions whose activity correlates with subjective values are thought to compose a brain valuation system (BVS), which mainly includes the ventromedial prefrontal cortex (VMPFC) and the ventral striatum (VS), sometimes accompanied by limbic regions (Haber and Knutson, 2010; Peters and Buchel, 2010). The BVS can express subject-specific values for various types of visual objects, in accordance with the common neural currency hypothesis (Chib et al., 2009; Lebreton et al., 2009). Here we suggest that observing 
Table 1. Overview of the subjects, procedures, and results

\begin{tabular}{|c|c|c|c|c|c|}
\hline & Group 1 & Group 2 & Group 3 & Group 4 & Group 5 \\
\hline No. of subjects & 24 & 25 & 24 & 24 & 19 \\
\hline Male/female subjects (№.) & $12 / 12$ & $10 / 15$ & $12 / 12$ & $12 / 12$ & $11 / 8$ \\
\hline Age (years; mean \pm SD) & $24.1 \pm 1.2$ & $22.4 \pm 2.6$ & $24.3 \pm 3.9$ & $22.4 \pm 3.2$ & $23.9 \pm 4.0$ \\
\hline No. of object pairs & 60 & 60 & 60 & 120 & 120 \\
\hline MDE (Z-score \pm SEM) & $0.13 \pm 0.07$ & $0.13 \pm 0.03$ & $0.12 \pm 0.06$ & $0.14 \pm 0.05$ & $0.18 \pm 0.04$ \\
\hline$t$ value & $t_{(23)}=1.88$ & $t_{(24)}=3.82$ & $t_{(23)}=1.97$ & $t_{(23)}=2.67$ & $t_{(18)}=4.28$ \\
\hline$p$ value & $<0.05$ & $<0.001$ & $<0.05$ & $<0.01$ & $<0.001$ \\
\hline Recognition task & No & No & No & Yes & Yes \\
\hline fMRI & No & No & No & No & Yes \\
\hline
\end{tabular}

someone directing an action toward an object affects the values encoded in the BVS.

Thus, our prediction was that MNS activity influences BVS activity, such that action observation modulates desirability ratings.

\section{Materials and Methods}

\section{Subjects}

The study was approved by the Ethics Committee for Biomedical Research of the Pitié-Salpêtrière Hospital (Paris, France), where it was conducted. A total of 116 young healthy subjects were included in the study after they gave written informed consent. Exclusion criteria were age younger than 18 years or older than 39 years, regular use of drugs or medications, history of psychiatric or neurological disorders, and contraindications to MRI (pregnancy, claustrophobia, or metallic implants). We first conducted behavioral studies in four groups of subjects $(n=24,25,24,24)$ recruited at the Cité Universitaire Internationale de Paris. We then recruited 19 subjects through the Relais d'Information sur les Sciences de la Cognition website for fMRI experiments. One of them was discarded because of technical problems during image acquisition, leaving 18 subjects for $\mathrm{fMRI}$ data analysis. Table 1 shows the demographic data.

\section{Stimuli}

A total of 120 different object pairs (e.g., food, toys, clothes, and tools) were selected to build the stimuli set. The two objects within a pair differed only by color (e.g., green and yellow; otherwise identical candies). For each object pair, two versions were filmed. In version A, one object (e.g., the green candy) was the goal of an action while the other (e.g., the yellow candy) was not, and vice versa for version B. Videos in which the object represents a goal $(\mathrm{G})$ constitute the $\mathrm{G}$ condition, and the other videos constitute the nongoal (NG condition) (Fig. 1). Thus, four videos were made for each pair of objects (i.e., two colors $\times$ two conditions). The duration was uniformly distributed between 2 and $5 \mathrm{~s}$ (mean \pm SEM: $3.546 \pm 0.932 \mathrm{~s}$ ). The same duration was used for the two differently colored objects of each pair. The size of the video frame on the computer screen was $640 \times 480$ pixels. All objects were also featured in high-resolution pictures $(436 \times 326$ pixels $)$ for the rating and recognition task.

In the $\mathrm{G}$ videos, objects were reached at, and sometimes grasped and moved by the agent. To avoid mere suggestion effects related to facial expressions, the upper part of the videos was kept out of frame such that the face of the agent was not visible. Not revealing the face also had the advantage of eliminating gaze processing and potential joint attention effects (Bayliss et al., 2006). The NG videos produced for the first behavioral studies included only static objects, on the same background as in $\mathrm{G}$ videos, and not including a human agent. This G versus NG comparison was implemented because it was consistently reported to activate the mirror neuron system (Rizzolatti and Sinigaglia, 2010). Because this basic comparison successfully demonstrated the predicted effects on desirability ratings, we included further controls. First, subjects may prefer G objects because of some movement in the video. To control for this first confound, we made NG videos in which objects were moving due to natural forces such as wind or gravity. Second, subjects may prefer $G$ objects because a human agent was present in the video, independently from the action performed. To control for this second confound, we made NG videos in which an agent was present and not acting upon the object (i.e., keeping still or moving around the object without acting upon it). In the end, $50 \%$ of NG videos included no movement and no agent, 25\% included moving objects, and 25\% included a human agent. By definition, all $\mathrm{G}$ videos contained a human agent; the object was moved in $\sim 33 \%$ of them and remained static in the other $67 \%$.

Tasks

All tasks were programmed on a PC using the Cogent 2000 (Wellcome Trust Centre for NeuroImaging, London, UK) library of MatLab (MathWorks) functions for stimuli presentation. We implemented two tasks, a rating (test) task and a recognition (control) task. Subjects in the first three groups were tested only on the rating task, which included 120 videos (60 object pairs). New videos were filmed for each behavioral study, including additional controls (as described). For the fourth and fifth behavioral studies, we grouped all further controlled videos such that subjects were shown a total of 240 videos (i.e., 120 object pairs). All experiments were divided into sessions comprising 60 videos, with different object pairs randomly distributed over the various sessions. The two objects of a pair always appeared in the same session to limit the effects of temporal fluctuations and of sessionwise rating scale anchors. In addition, the presentation order of the different videos was randomized for each subject, with the constraint that the first object and the second object of each pair should appear in the first half and the second half of a session, respectively. To eliminate color preferences at the group level, half the subjects were administered version $\mathrm{A}$ and the other half version $B$ of the task. This ensured that, for example, the green candy was the G object for half the subjects, but was the NG object for the other half, and vice versa for the yellow candy.

Instructions given for the rating task slightly varied over the successive behavioral studies. More specifically, subjects were asked to state "how much they like the object" (study 1), "how much they would like to use the object" (study 2), or "how much they would like to acquire the object" (studies 3-5). Questions were focused on objects because we wanted to assess whether observing object-directed actions increases the value of the object, not the propensity to imitate the action. The key manipulation (that objects were presented in G/NG pairs) was never mentioned to the subjects.

Every trial of the rating task started with a fixation cross displayed for $1.5 \mathrm{~s}$ and was immediately followed by the video, which lasted between 2 and $5 \mathrm{~s}$ (Fig. 1A). Next, the desirability scale appeared on the screen below the picture of the object to be rated (without human agent). The scale was graduated from 0 (not desirable) to 10 (highly desirable). Subjects could move the cursor by pressing a button with their right index finger to go left or with their right middle finger to go right. Rating was self-paced: subjects had to press a button with their left index finger to validate their response and proceed to the next trial. The initial cursor position on the scale was randomized to avoid confounding the ratings with the movements they involved. In the fMRI experiment, mean response time was $2888 \pm 254 \mathrm{~ms}$. The total trial duration was therefore almost $8 \mathrm{~s}$ on average $(1500 \mathrm{~ms}$ of fixation $+3500 \mathrm{~ms}$ of video $+2888 \mathrm{~ms}$ of rating).

The recognition task was introduced in the fourth behavioral study to control for attentional effects. Indeed, it might be argued that G objects are more salient because they appear as the goals of actions. This is not necessarily intuitive because one could argue, on the contrary, that an 
A RATING TASK (version A)

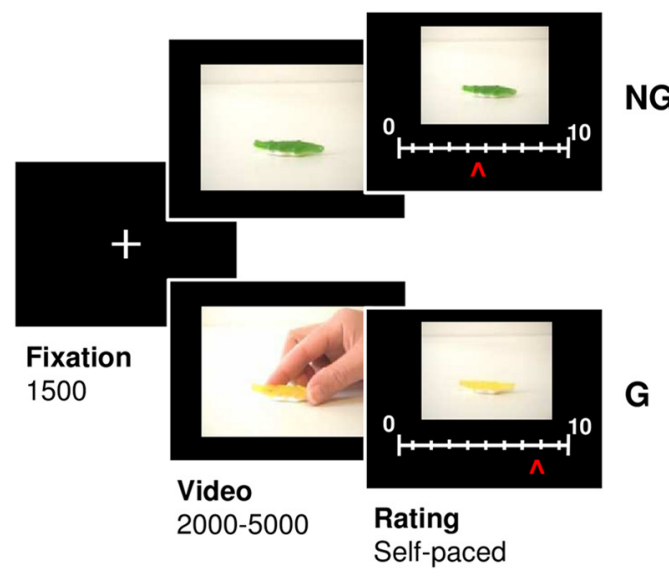

RATING TASK (version B)

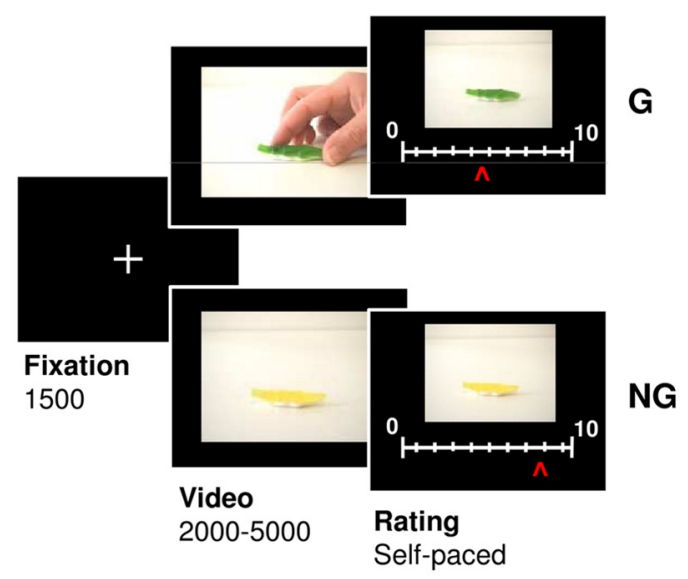

B RECOGNITION TASK
Choice

Self-paced

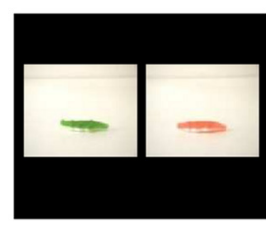

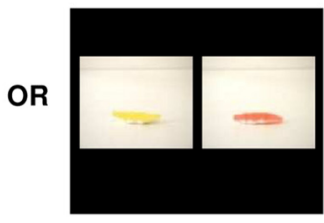

Choice

Self-paced
Figure 1. Behavioral tasks. $A$, The desirability rating task. Successive screens displayed in one trial are shown from left to right with durations in milliseconds. Subjects had to rate the object featured in the video by moving a cursor along an analog scale. The object was taken as the goal of an action in the $G$ condition but not in the NG condition. Each object had an identical, yet differently colored, counterpart with which it formed a pair. Within each pair, one object was featured in a $G$ video and the other in an NG video. The two objects of a pair were always presented in the first and second halves of the same experimental session. Between the two versions of the task ( $A$ and $B$ ), the conditions ( $G$ and $N G$ ) assigned to the two objects were swapped. In the example illustrated, the green candy was the $G$ object in version $A$ but the NG object in version $B$, and vice versa for the yellow candy. To eliminate color preferences at the group level, half the subjects performed version $A$ and the other half performed version B. B. The recognition task. Subjects had to select the "old" object, which meant the object that had been featured in the videos (either $G$ or NG) shown during the rating task. Every choice contained one old and one "new" object. In the illustrated example, the correct answer would be green for the choice on the right and yellow for the choice on the left. object moving by itself, or ignored by the action, would grab more attention. We reasoned that if they grab more attention, G objects should be better encoded in memory and therefore better recognized at the end of the experiment. To test this possibility, we presented 80 pairs of pictures to the subjects in an old/new recognition task. For each pair, one picture contained an object that subjects had seen during the rating task (in a $G$ or an NG video), and the other picture contained the same object with a third and previously unseen color (Fig. $1 B$ ). The 80 pairs of pictures were fixed, but the order of their presentation was randomized for every subject. The two pictures of a pair were displayed side by side after a $500 \mathrm{~ms}$ fixation cross. The relative position of the two pictures on the screen was also randomized. Subjects were asked to select the picture they had already seen (the old one). The task was self-paced; subjects used their index/middle finger to choose the left/right picture and proceed to the next trial.

The rating and recognition tasks used in the fMRI experiment were identical to those used in the fourth behavioral study, which included 240 videos with controls for the occurrence of movement and the presence of a human agent. Desirability ratings were neutral on average (mean, 4.91) but extended over a wide range along the scale (SD, 2.41). The interobject SD (2.41) was similar to the intersubject SD (2.51), indicating that, just as each subject used a large range of values to rate the different objects, a same object received a large range of ratings by the different subjects.

After fMRI scanning, subjects were asked to complete an Empathy Questionnaire (EQ) and to perform the Reading Mind in the Eyes test. These measures were taken because they respectively assess empathizing and mentalizing abilities, which might relate to the goal contagion effect that we intended to demonstrate (Baron-Cohen, 2009). At the end of the experiments, subjects were debriefed to assess their knowledge about the task structure and their awareness of the mimetic desire effect (MDE).

For statistical analysis, desirability ratings were $Z$-scored at the session level for each subject. The MDE was defined as the difference in $Z$-scored ratings between $\mathrm{G}$ and $\mathrm{NG}$ objects. Performance on the recognition task was expressed as the percentage of errors. For both tasks, $\mathrm{G}$ and NG conditions were compared at the group level using one-tailed paired $t$ tests. Correlations between demographic and behavioral variables were assessed using robust regression tests. We considered three significance levels: $0.05,0.01$, and 0.001 . All statistical analyses were performed with the MatLab Statistical Toolbox (MatLab R2006b; MathWorks).

\section{Neuroimaging}

Data acquisition. $\mathrm{T} 2{ }^{*}$-weighted echoplanar images (EPIs) were acquired with blood oxygenation level-dependent (BOLD) contrast on a 3.0 tesla magnetic resonance scanner (Trio; Siemens). We used a tilted plane acquisition sequence designed to optimize functional sensitivity in the orbitofrontal cortex and medial temporal lobes (Deichmann et al., 2003; Weiskopf et al., 2006). To cover the whole brain with good spatial resolution, we used the following parameters: $\mathrm{TR}=2.0 \mathrm{~s}, 35$ slices, $2 \mathrm{~mm}$ slice thickness, $1.5 \mathrm{~mm}$ interslice gap. T1-weighted structural images were also acquired, coregistered with the mean EPI, normalized to a standard T1 template, and averaged across subjects to allow group-level anatomical localization. EPI data were analyzed in an event-related manner, within a general linear model, using the statistical parametric map (SPM) software (SPM5) (Wellcome Trust Center for NeuroImaging, London, UK) implemented in MatLab. The first five volumes of each session were discarded to allow for T1 equilibration effects. Preprocessing consisted of spatial realignment, normalization using the same transformation as structural images, and spatial smoothing using a Gaussian kernel with a full-width at half-maximum (FWHM) of $8 \mathrm{~mm}$. To correct for motion artifacts, subject-specific realignment parameters were included in all general linear models (GLMs) as covariates of no interest.

Neural activation. We used two GLMs to explain individual-level functional scans. All models incorporated two events per trial (videoviewing and object-rating periods), which were modeled by boxcar functions. In the first model (GLM1), video viewing was modeled with two separate regressors for the two main conditions ( $G$ and NG). Each of these regressors was parametrically modulated by binary indicators of whether the object was presented first or second in the pair, whether the 
A
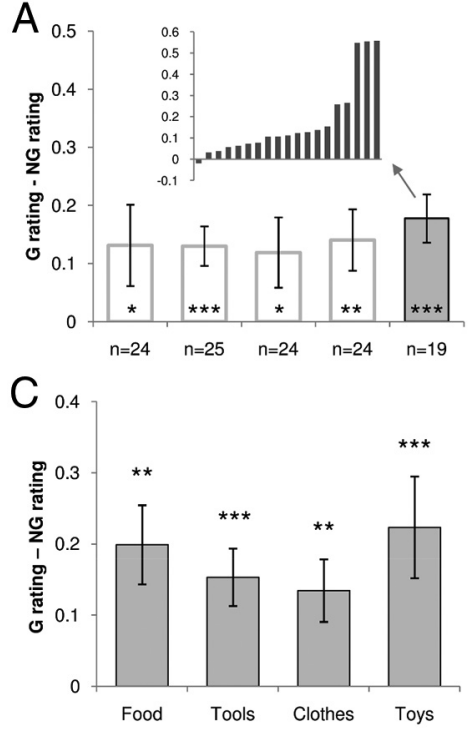

B

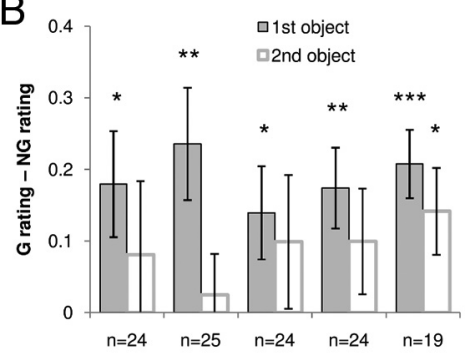

D

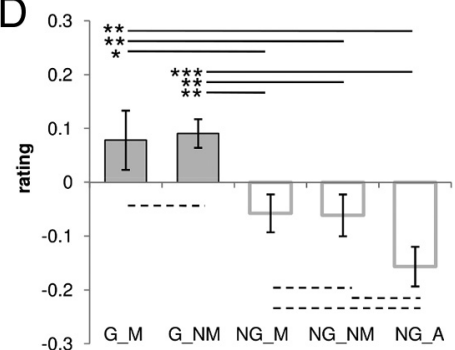

$E$
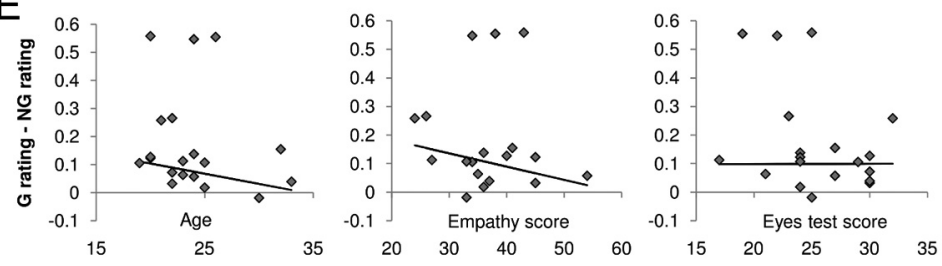

Figure 2. The MDE. Histograms illustrate the MDE, defined as the difference in Z-scored desirability ratings between $G$ and NG objects. Error bars indicate intersubject SEM. ${ }^{*} p<0.05,{ }^{* *} p<0.01,{ }^{* * *} p<0.001$; paired $t$ test. $A$, Overall MDE obtained in the five groups of participants. Group 5 (filled bar) corresponds to the subjects who were scanned using fMRI while performing the task. MDE of individual subjects in this group are shown above the bars. $\boldsymbol{B}, \mathrm{MDE}$ calculated separately for the two objects of a pair. Filled and empty bars indicate objects that were presented during the first and second parts of an experimental session, respectively. $\boldsymbol{C}$, MDE obtained in Group 5 for the different types of objects presented in the videos. D, MDE obtained in Group 5 for the different categories of videos. $G$ videos (filled bars on the left) contained objects that were moved (G_M) or not (G_NM). NG videos (empty bars on the right) contained a human agent (NG_A) or not, with the object moved (NG_M) or not (NG_NM). As indicated with asterisks, all comparisons between $G$ and NG categories were significant (solid lines), whereas no significant difference was found between G or NG subcategories (dashed lines). $\boldsymbol{E}$, Correlation between MDE and other variables (age, empathy, and eye test scores). The $y$-axis indicates the MDE (difference in Z-scored desirability rating between G and NG objects). Scatter plots show the MDE for all individuals. No correlation was significant (tested using robust regression).

object was moved or not moved, whether a human agent was present or not present (for NG condition only), and the desirability ratings attributed to the objects. The object-rating period was modeled in a single regressor, parametrically modulated by response time. In the second model (GLM2), the G and NG conditions were modeled in the same regressor, one for video viewing and one for object rating. The objectrating period was again modulated by response time. The video-viewing regressor was modulated with six parameters: the same three binary indicators (order, movement, and agent) as in GLM1, the condition (coded 0 for NG and 1 for G), the average rating (over the two objects of a pair), and the differential rating (difference between current and average ratings). Parametric modulations were included in this order and sequentially orthogonalized by SPM software. The regression coefficient $(\beta)$ obtained for differential rating therefore did not merely reflect action observation (G vs NG condition) or overall preference (for a particular object). All regressors of interest were convolved with a canonical hemodynamic response function (HRF) and its first temporal derivative.

Linear contrasts of regression coefficients were computed at the subject level and then taken to a group-level, random-effect analysis using one-sample $t$ tests. We only analyzed the $\beta$ obtained for the canonical HRF, discarding the temporal derivative. All activations reported in the main text survived a threshold of $p<0.05$ after clusterwise familywise error (FWE) correction for multiple comparisons over the entire brain (minimum of 65 voxels). Regions of interest (ROIs) were identified in SPMs at a voxelwise threshold of $p<0.001$ (uncorrected for multiple comparisons) and a clusterwise threshold of $p<0.05$ (FWE corrected for multiple comparisons). ROIs were selected on the basis of existing literature about the MNS and BVS. For both brain systems, we restricted our analyses to the left hemisphere, which contained significant maxima for all ROIs at the group level. To further characterize the activity in our ROIs, we extracted the $\beta$ within $8 \mathrm{~mm}$ diameter spheres (corresponding to the FWHM of the Gaussian kernel used for spatial smoothing). The spheres were centered on the voxels that were inside the ROIs and maximally activated at the group level by the relevant contrast (i.e., the contrast between $\mathrm{G}$ and NG video-viewing periods for the MNS ROIs and the parametric modulation by desirability ratings for the BVS ROIs). Regression coefficients were averaged within ROIs for each subject and then tested for significance at the group level using onesample $t$ tests.

To extract deconvolved response magnitudes for all videos, we estimated a third model that contained a regressor for every trial. These regressors included a boxcar over the videoviewing period, convolved with a canonical HRF and its first temporal derivative. Then $\beta$ s obtained for the canonical HRF were extracted from the same ROIs described above. To compensate for intersession and interindividual differences, these trial-by-trial $\beta$ s were $Z$-scored at the session level.

A Bayesian Model Selection (BMS) procedure was used to compare goodness of fit between alternative GLMs. The main purpose of this analysis was to assess the relevance of breaking down desirability ratings into average and differential ratings. Note that average and differential ratings are strictly orthogonal because the two objects of a pair have the same average rating but opposite differential rating. Activity was fitted in the four main ROIs defined by appropriate GLM1 contrasts (G vs NG condition for MNS regions, modulation by ratings for BVS regions). SPM thresholds were adjusted to obtain large ROIs of approximately equivalent size: $\mathrm{PL}=366$ voxels, $\mathrm{PMC}=324$ voxels, $\mathrm{VS}=353$ voxels, $\mathrm{VMPFC}=578$ voxels. The model space explored in the BMS (see Fig. $4 B$ ) was composed of nine GLMs, each of which included one categorical regressor modeling the video-viewing period with a boxcar function, plus various combinations of parametric modulators. A first GLM contained solely the modulation by experimental condition ( 0 for NG, 1 for $\mathrm{G}$ ). Then we built three GLMs that differed on the parametric regressor accounting for desirability ratings: this parametric modulator was current object rating, average rating, or differential rating. A fifth GLM included two parametric modulations, one with average rating and one with differential rating. The last four GLMs were replicated with the addition of G/NG condition as the first parametric modulation, leading to a total of nine GLMs. These nine GLMs were estimated at the individual level for each ROI using variational Bayesian techniques, with spatial smoothness priors (Harrison et al., 2008). This estimation procedure provided a $\log$ evidence for each GLM and ROI. Log evidences were fed into a BMS random-effects analysis (Stephan et al., 2009), which computed the exceedance probability for each GLM in each ROI. This measure gives the probability that, within the model space, the considered GLM is the most likely given the activity recorded in the considered ROI.

Neural connectivity. Effective connectivity between MNS and BVS regions was analyzed using Dynamic Causal Modeling (DCM) procedures (Daunizeau et al., 2009). The GLM built for this analysis con- 
tained the following regressors: one boxcar function covering video-viewing periods, two parametric modulations of this function by the experimental condition ( $\mathrm{G}$ vs NG) and by desirability ratings, one boxcar function over video-rating periods, with parametric modulation by response time. All regressors were convolved with a canonical HRF. After GLM estimation using SPM8, the signal was extracted from $8 \mathrm{~mm}$ spheres centered on the session-specific individual maximum within the $8 \mathrm{~mm}$ sphere centered on the relevant group-level maxima (illustrated in SPMs) (see Fig. 3). These maxima were obtained for parametric modulation by G/NG condition for MNS regions (PL and PMC) and by desirability rating for BVS regions (VS and VMPFC). The signal was averaged across voxels within each ROI and adjusted for effects of no interest (everything except G/NG). Comparison between DCMs was principally meant to assess the likelihood of different network architectures (from parallel to serial) and different directions of information flow (between MNS and BVS regions). In all cases, the driving input was the G/NG modulation of the boxcar function covering video-viewing periods. To assess network architecture, nine DCMs were iteratively built by adding connections between nodes, starting from a full parallel and ending with a full serial network (see Fig. 5A). The first DCM corresponds to the G/NG manipulation directly affecting all ROIs in parallel and the last DCM to a serial caudorostral transfer of information, from MNS to BVS regions. To assess the direction of information flow, we built two additional serial DCMs, one with a reverse order from rostral to caudal regions and one in which MNS and BVS regions were swapped (see Fig. $6 A$ ). For both comparisons, the exceedance probability of the different DCMs was computed using standard BMS procedure as described (see Figs. 5B, 6B). We further checked that all the arrows of the winning model (both modulation and connectivity parameters) survived one-sample $t$ tests for group-level random effect, showing that means of posterior distributions were significantly different from zero.

To confirm DCM analysis of effective connectivity, we also used iterative multiple regressions, which allow including the behavioral response (desirability rating) in addition to experimental manipulation ( $\mathrm{G}$ vs NG) and brain activity. Multiple robust regressions between inputs, $\beta$ values, and outputs were performed across trials at the individual level. We used a series of GLMs that progressively included more nodes while keeping the previous nodes as covariates. We started by regressing the G/NG parameter (coded 1/0) against the $\beta$ values extracted by deconvolution in the PLs. Next, we regressed the PL against the PMC, controlling for the G/NG variable. Iteratively, we examined the regression of PMC against VS, then VS against VMPFC, and finally VMPFC against desirability ratings. At each step, we controlled for the variance explained by all previous variables $(\mathrm{G} / \mathrm{NG}, \mathrm{G} / \mathrm{NG}+\mathrm{PL}$, $\mathrm{G} / \mathrm{NG}+\mathrm{PL}+\mathrm{PMC}$, and $\mathrm{G} / \mathrm{NG}+\mathrm{PL}+\mathrm{PMC}+\mathrm{VS}$, respectively). Regression coefficients were estimated at the individual level and then tested for a grouplevel random effect using one-sample $t$ tests.
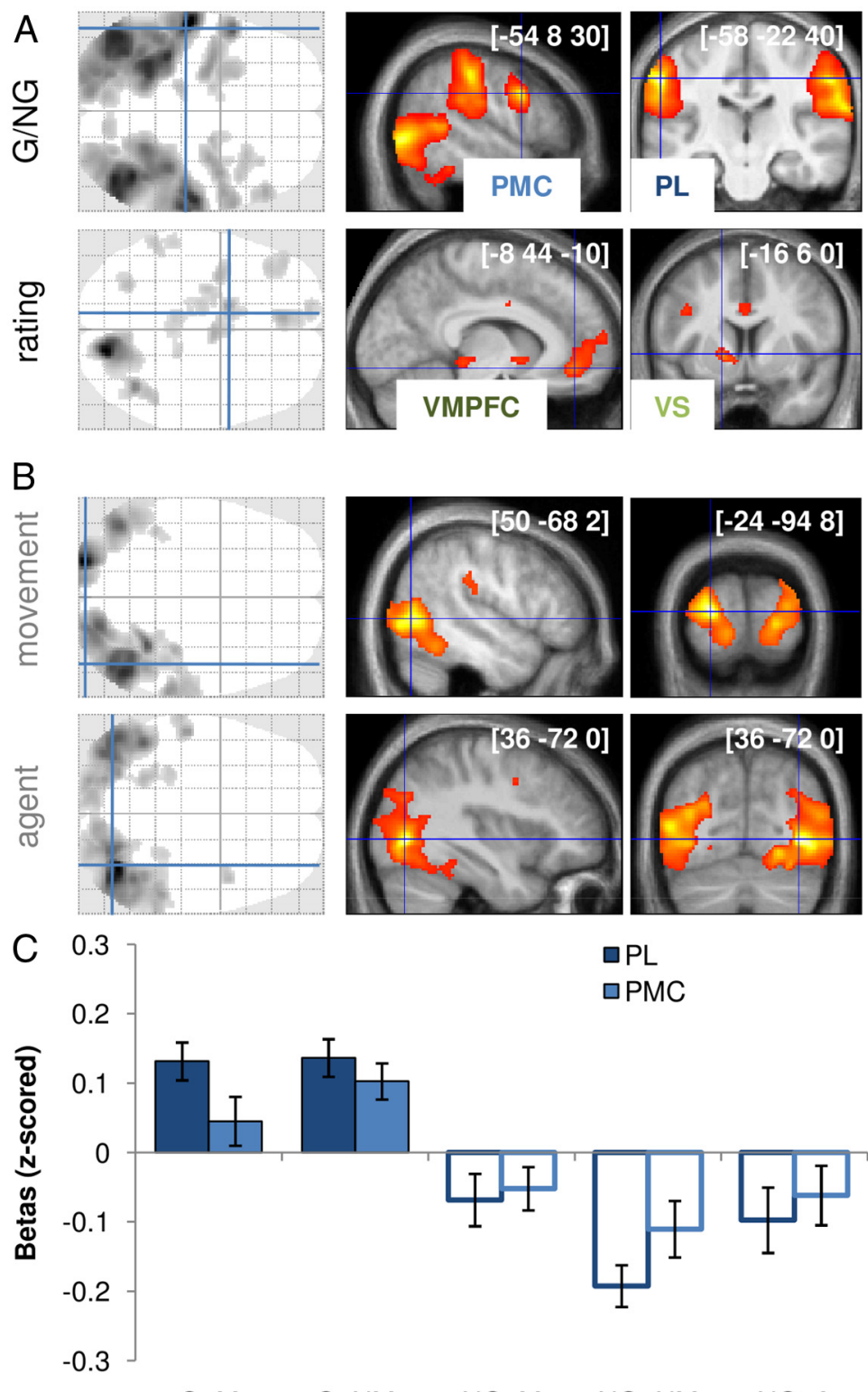

Figure 3. ROls isolated from group-level activations. SPMs were obtained using GLM1 (in Materials and Methods). $A$, Contrasts of interest. Top, MNS was isolated with the contrast between goal-directed and nongoal-directed actions (G and NG videos). Bottom, BVS was isolated with the parametric modulation by desirability ratings. B, Contrasts of no interest. Top, Occipitotemporal regions activated during videos presenting some movement. Bottom, Occipitotemporal regions activated during NG videos featuring a human agent. Areas shown in gray/black on glass brains and in red/yellow on slices showed significant group-level random effect ( $p<0.05$ after correction for multiple comparisons at the cluster level). The $[x, y, z]$ coordinates of the different maxima refer to the Montreal Neurological Institute (MNI) space. Slices were taken in the different ROIs, along planes orthogonal to the lines indicated on glass brains. Blue, MNS; green, BVS. C, MNS activations presented separately for the different categories of videos. Regression coefficients were estimated by deconvolution of the hemodynamic response to video display and extracted from ROIs centered on PMC and PL group-level maxima. G videos (filled bars on the left) contained objects that were moved (G_M) or not moved (G_NM). NG videos (empty bars on the right) contained a human agent (NG_A) or not, with the object moved (NG_M) or not (NG_NM). For both regions, all comparisons between NG and $\mathrm{G}$ categories remained significant or bordered significance (all $p<0.06$, paired $t$ test). Error bars indicate intersubject SEM.

We last explored individual differences in the susceptibility to MDE. We first used a group-level GLM to identify brain regions in which the G/NG contrast was correlated across subjects with the observed MDE magnitude. Subjects with big versus small MDE were sorted using a median split. GLM contained not only this factor (1 for big MDE, 0 for small MDE) but also age, sex, and response time as covariates of no interest. Regression coefficients were then extracted by deconvolution from the ROI (the VS) activated in this analysis, contrasted between G 
and NG conditions, and tested for correlation with MDE magnitude using direct robust regression. We also built a GLM including connectivity measures obtained for all forward links of the most probable DCM to predict individual variations in VS response to the G/NG manipulation. All GLM estimations and robust regression tests were performed using the MatLab Statistical Toolbox (MatLab R2006b; MathWorks).

\section{Results}

\section{Behavioral data}

To demonstrate the phenomenon of goal contagion, we asked a total of 116 volunteers to rate the desirability of objects featured in short videos (Table 1 shows an overview of experimental procedures). Results indicated a significant MDE, or higher ratings for $\mathrm{G}$ than for $\mathrm{NG}$ objects $\left(5.13 \pm 2.37\right.$ vs $4.69 \pm 2.44 ; t_{(115)}=$ $8.50 ; p<0.001$, paired $t$ test). Thus, the same object was considered more desirable when taken as a goal by another agent. At debriefing, most subjects reported the existence of object pairs (the two colors) and conditions (G vs NG) but could not tell whether they preferred $\mathrm{G}$ or NG objects, suggesting that the MDE was largely implicit. The MDE was significant in all participant groups (all $p<0.05$, paired $t$ test), indicating that it was not crucially sensitive to the precise formulation of the question (Fig. $2 A)$. In all groups, the MDE was greater when considering solely the object presented first for each pair (i.e., before color counterbalancing with the presentation of the second object) (Fig. $2 B$ ). Similar MDEs were obtained for the various kinds of objects used in the videos (food, tools, clothes, toys) and for both sexes (Fig. 2C). There was no significant correlation between the MDE and participant age (although younger people tended to show greater MDE) and no correlation with empathy or eye test scores (Fig. 2E).

In the first group, NG videos contained only a static object and no human agent. For the second and third groups, we progressively included further controls to rule out potential confounds (see Materials and Methods). Results (Fig. 2D) showed that G objects remained significantly more desirable than NG objects in all situations (all $p<0.05$, paired $t$ test). Therefore, the MDE occurred regardless of whether $\mathrm{G}$ objects were moved; importantly, this occurred even when compared with NG objects moved or located close to a human agent. To further address the possibility of attentional confounds, we added a recognition task at the end of our experimental procedure for participant Groups 4 and 5. The error rate was similar for G and NG objects ( $\sim 15 \%)$, indicating that $\mathrm{G}$ objects were no better encoded in memory than NG objects. Furthermore, the objects correctly recognized received desirability ratings similar to those that were not recognized. Thus, there was no reason to believe that ratings were affected by an attentional bias favoring the G or the NG condition. Note, however, that retrospective assessment might not be sensitive enough to pick up transient arousal occurring during the task. To assess the influence of the different confounding factors on the desirability ratings of fMRI participants (Group 5), we estimated a GLM including presentation order (1 or 2), occurrence of movement ( 0 or 1 ), presence of an agent ( 0 or 1$)$, and response time in addition to the G/NG manipulation (coded $1 / 0)$. The regression coefficient obtained for each factor was entered into a group-level random-effects analysis using onesample $t$ tests. The G/NG factor was the only significant predictor of desirability rating $\left(\beta=0.15 \pm 0.04 ; t_{(18)}=3.62 ; p<0.01\right)$ when correcting for multiple tests (dividing the threshold by five).
Table 2. List of activations in the G/NG contrast from GLM1

\begin{tabular}{lccc}
\hline & $\begin{array}{l}\text { MNI coordinates } \\
(\mathrm{mm})\end{array}$ & $\begin{array}{l}\text { Cluster size } \\
\text { (voxels) }\end{array}$ & $\begin{array}{l}\text { Peakt } \\
\text { value }\end{array}$ \\
\hline $\begin{array}{l}\text { Region } \\
\text { Occipito-temporo-parietal cortex }\end{array}$ & & & \\
$\quad$ Right & $44,-62,2$ & 12,512 & 14.95 \\
$\quad$ Left & $-58,-22,40$ & 14,686 & 17.31 \\
$\begin{array}{l}\text { Premotor cortex } \\
\quad \text { Right }\end{array}$ & $42,0,62$ & 1017 & 7.54 \\
$\quad$ Left & $-54,8,30$ & 1426 & 11.41 \\
Insula & $-36,-6,12$ & 77 & 6.89 \\
Inferior frontal gyrus & $54,32,2$ & 254 & 6.80 \\
Amygdala & & & \\
$\quad$ Right & $20,-6,-18$ & 308 & 6.41 \\
$\quad$ Left & $-26,6,-28$ & 735 & 6.22 \\
Cerebellum & & & \\
$\quad$ Right & $10,-78,-48$ & 178 & 5.42 \\
$\quad$ Left & $-10,-80,-50$ & 155 & 6.29 \\
Thalamus & $-16,-30,0$ & 95 & 5.48 \\
\hline
\end{tabular}

$p<0.05$, whole-brain, clusterwise FWE correction.

Table 3. List of activations in the rating contrast from GLM1

\begin{tabular}{lccc}
\hline Region & $\begin{array}{l}\text { MNI coordinates } \\
(\mathrm{mm})\end{array}$ & $\begin{array}{l}\text { Cluster size } \\
\text { (voxels) }\end{array}$ & $\begin{array}{r}\text { Peakt } \\
\text { value }\end{array}$ \\
\hline $\begin{array}{l}\text { Lingual gyrus } \\
\quad \text { Right }\end{array}$ & $14,-80,0$ & 2013 & 14.54 \\
$\quad$ Left & $-8,-32,-6$ & 132 & 4.67 \\
Cerebellum & $40,-60,-44$ & 201 & 7.83 \\
Ventral striatum + thalamus & $-16,6,0$ & 353 & 6.74 \\
Ventromedial prefrontal cortex & $-8,44,-10$ & 578 & 6.12 \\
Median cingulate & $2,-4,32$ & 160 & 6.06 \\
Paracingulate gyri & $-2,-34,36$ & 66 & 4.29 \\
Precentral gyrus & $-38,0,30$ & 159 & 5.70 \\
Middle temporal gyrus & & & \\
$\quad$ Right & $42,-52,-16$ & 122 & 5.34 \\
$\quad$ Left & $-56,-44,-12$ & 145 & 4.87 \\
Middle occipital gyrus & $-30,-66,28$ & 114 & 5.28 \\
Parahippocampal gyrus & $-28,-8,-34$ & 71 & 5.00 \\
Middle + superior frontal gyrus & $-42,34,18$ & 333 & 4.89 \\
Supramarginal + angular gyri & $-58,-32,44$ & 86 & 4.49 \\
\hline
\end{tabular}

$p<0.05$, whole-brain, clusterwise FWE correction.

\section{Neuroimaging data}

To investigate the mechanisms by which viewing goal-directed actions can possibly influence desirability ratings, participants in Group 5 were scanned using fMRI while they performed the last version of the rating task, which included all control videos. We analyzed brain activity during the video-viewing period, which we modeled as a boxcar convolved with the canonical HRF. In GLM1, two regressors were included (for G and NG conditions), and each of them was parametrically modulated by subjective ratings and factors of no interest (see Materials and Methods). All SPMs shown hereafter (Fig. 3) were thresholded at $p<0.05$ after clusterwise FWE correction for multiple comparisons over the entire brain.

The direct contrast between $\mathrm{G}$ and NG conditions activated a large brain network encompassing visual occipitotemporal pathways, parietal lobules, and premotor cortical areas (Fig. 3A; Table 2). Given our a priori assumptions about the MNS, we focused subsequent analyses on spheres positioned over PL and PMC regions. In particular, the superior temporal sulcus was discarded because it is involved in action observation but not in action execution (Iacoboni and Dapretto, 2006; Van Overwalle and Baetens, 2009; Rizzolatti and Sinigaglia, 2010). The correlation with subjective ratings delineated another set of brain re- 
gions, including the ventral visual pathway, the ventral frontostriatal regions, and the midline cingulate cortical areas (Fig. 3A; Table 3). Again, based on our a priori assumptions about the BVS, we focused subsequent analyses on VS and VMPFC activation foci. In particular, midline cingulate areas are not classically cited as components of the BVS (Rangel et al., 2008; Haber and Knutson, 2010; Peters and Buchel, 2010).

We checked that the different confounding factors were not significantly impacting our main ROIs. In fact, they activated different brain regions (Fig. 3B): movement occurrence and agent presence were reflected primarily in occipital and temporal regions (including the superior temporal sulcus). We then deconvolved the BOLD signal by fitting a canonical HRF to every video-viewing period (see Materials and Methods). From this, we obtained regression coefficients ( $\beta$ values) that estimated response magnitudes to all objects in our main ROIs. Response magnitude in the PL and PMC was positive for all types of $\mathrm{G}$ videos (regardless of whether the object was moved) and negative for all NG controls (regardless of whether movement and agent were present). All comparisons between $\mathrm{G}$ and NG subcategories were significant or bordering significance (all $p<0.06$, one-sample $t$ test).

This first analysis (using GLM1) confirmed that $G$ versus NG contrast reveals increased activity in regions known to be involved in observing goal-directed actions (PL, PMC) and that subjective ratings correlate with activity in regions known to be involved in valuating objects (VS, VMPFC). We next examined, with GLM2, which of these regions would capture the MDE. In GLM2, we broke down subjective ratings into two orthogonal variables: the average with the rating of the paired object and the difference from this average. Average ratings indexed the desirability of the object regardless of the condition ( $\mathrm{G}$ or NG), that is, the preexistent preference for this object. Differential ratings indexed the impact of our manipulation ( $\mathrm{G}$ vs NG) on the object de-

sirability, that is, the MDE obtained trial by trial. As expected (Fig. $4 A$, top), average ratings were significantly expressed in BVS regions (VS, VMPFC; both $p<0.01$, one-sample $t$ test) but not in MNS regions (PL, PMC). Crucially, correlation with differential ratings was significant in PMC, VS, and VMPFC (all $p<$ 0.05 , one-sample $t$ test). Thus, when moving from MNS to BVS regions, the representation of $\mathrm{G} / \mathrm{NG}$ information tended to vanish whereas that of differential rating progressively emerged. The PMC seems central in this process because it was the only region activated (Fig. $4 A$, bottom) in the conjunction between $\mathrm{G} / \mathrm{NG}$ and differential rating parametric modulators (at a threshold of $p<0.001$, uncor-
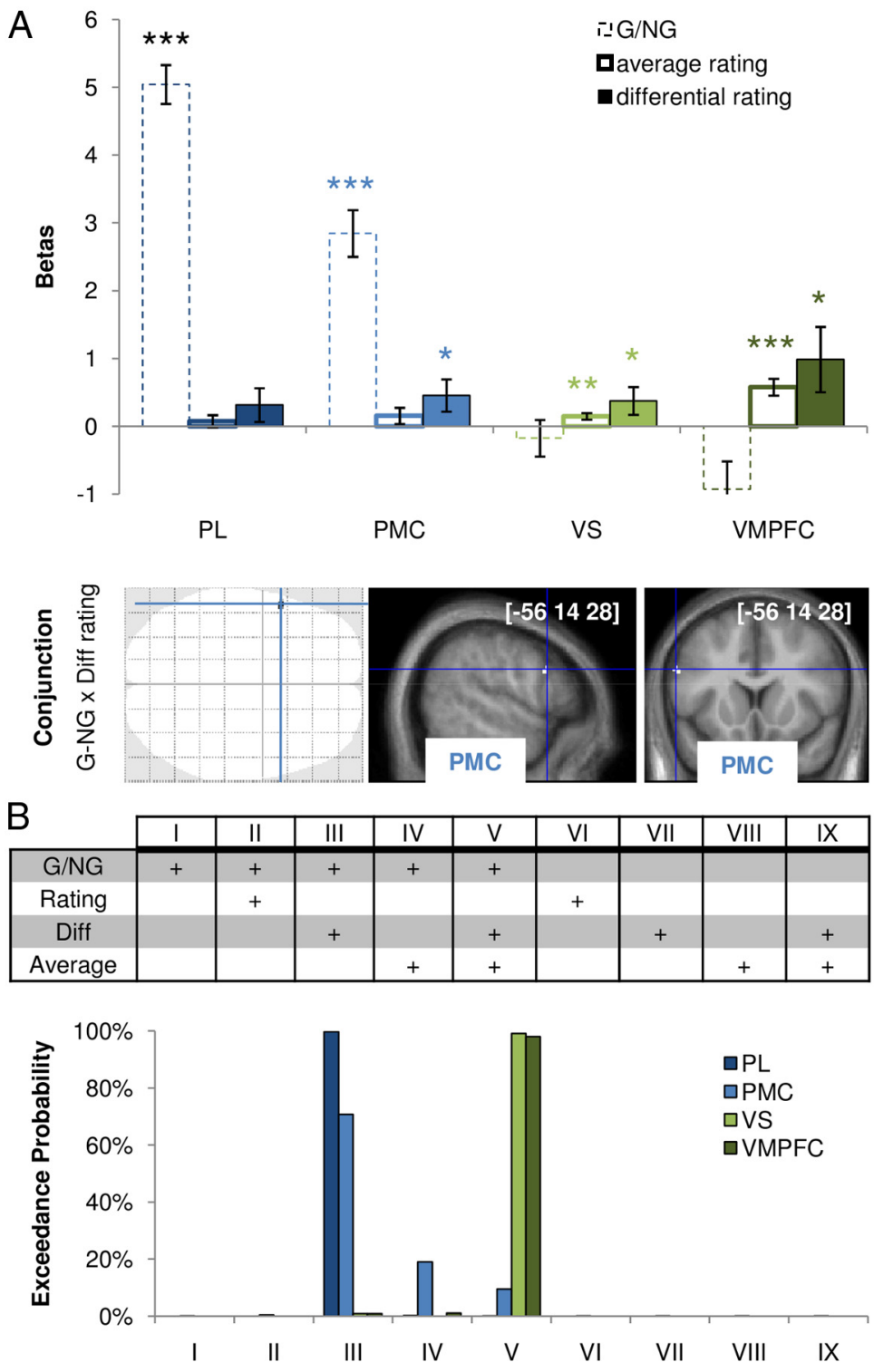

Figure 4. Neural correlates of the MDE. A, Information encoded in the different ROls. Regression coefficients were estimated using GLM2 parametric modulators. G/NG (dotted bars): binary variable that signaled objects being taken as goals. Average rating (empty bars): mean rating over current and paired objects. Differential rating (filled bars): difference between current object and average rating. Error bars indicate intersubject SEM. ${ }^{*} p<0.05,{ }^{* *} p<0.01,{ }^{* * *} p<0.001$; one-sample $t$ test. SPM was obtained from the conjunction between $\mathrm{G} / \mathrm{NG}$ contrast and modulation by differential rating. Areas shown in black on glass brains and in white on slices showed significant group-level random effect ( $p<0.001$, one-sample $t$ test; uncorrected, minimum of 10 voxels). Sagittal and coronal slices were taken at the global maximum of interest (in the premotor cortex). The $[x, y, z]$ coordinates of the er to the Montreal Neurological Institute space. $\boldsymbol{B}$, Comparison between GLM in the different ROls. Bars indicate the probability of different models estimated using BMS. The model space explored in the BMS is illustrated in the table above the bars. Models could include or not include the G/NG contrast and could decompose or not decompose the desirability ratings into differential (Diff) and average ratings. + , Regressor included in the GLM.

rected). BMS analysis confirmed the explanatory power of decomposing desirability ratings into average and differential ratings. Indeed, among the different GLMs tested (see Materials and Methods), PL and PMC activity was better fitted with G/NG and differential rating as regressors of interest, whereas VS and VMPFC activity was better accounted for using three regressors: $\mathrm{G} / \mathrm{NG}$, average, and differential rating (Fig. $4 B$ ). Thus, this analysis further supports the idea that the MDE (as indexed by differential ratings) involved the participation of both MNS and BVS regions, whereas pre-existent preferences for the different objects (as indexed by average ratings) were represented in BVS but not in MNS regions. 


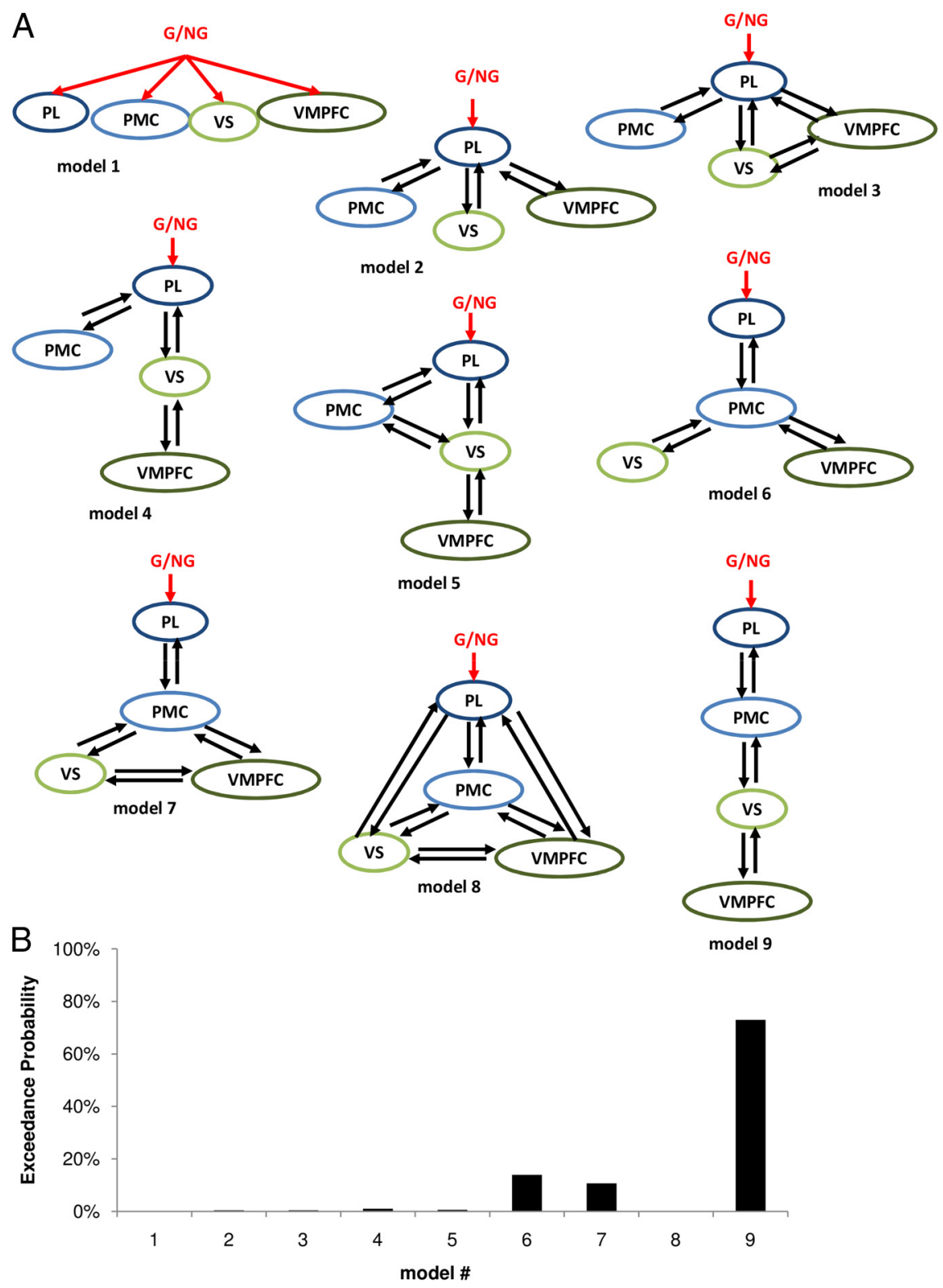

Figure 5. Network architecture. $\boldsymbol{A}$, Illustration of alternative DCMs. In all models, the driving input (red) was a boxcar function over the video-viewing period, parametrically modulated by the experimental condition ( 1 for goal, 0 for nongoal objects). The different models account for different levels of branching, from a full parallel (model 1) to a serial caudorostral transfer of information (model 9). $\boldsymbol{B}$, Results of the BMS procedure used to identify the most probable model.

We next examined effective connectivity, using DCM analysis, to understand how G/NG information could be translated through MNS and BVS networks into desirability ratings. Our hypothesis was that MNS regions, which are activated when observing goal-directed actions, modulate BVS regions, which express desirability ratings. A natural implementation of this hypothesis is a caudorostral transfer of information between inputs and outputs. We first questioned the degree of branching in the network, testing all intermediate possibilities between our fully serial hypothesis and a fully parallel network, in which the G/NG input would be processed by the different ROIs independently (Fig. 5A). BMS analysis identified the fully serial model as the most probable (Fig. $5 B$ ). We then questioned the directionality of information flow, trying other models in which the G/NG input was processed by BVS regions before affecting MNS activity (Fig. 6A). The most probable model was again the fully serial caudorostral architecture (Fig. 6B). We checked that the individual means of posterior distributions were significantly different from zero (all $p<0.05$ ) at the group level for all links of the winning model, including the forward link from PMC to VS (Fig. $6 A$, left model). Interestingly, when VS and VMPFC positions were swapped, the connection from PMC to VMPFC was found to be not significant, indicating that this connection is not likely to represent a path from MNS to BVS. We also note that all models in which G/NG information could reach the BVS without passing through the PMC (models 1-5, 8, B, C) had negligible exceedance probabilities $(<1 \%)$. These observations suggest that the PMC-VS forward connection is the way for the MNS to influence the BVS. Finally, we envisaged less parsimonious models in which not only the input region but also one unidirectional or bidirectional connection was modulated by the G/NG manipulation. They were discarded because none of them could prove more probable than the straight serial model (all exceedance probabilities being $<20 \%$ ). In other words, model connections enabled the G/NG manipulation to influence desirability ratings but were not themselves sensitive to this manipulation. Thus, comparison of alternative DCMs supported our hypothesis that the MDE is underpinned by MNS-mediated modulation of BVS response.

We then assessed whether this hypothesis could also explain intersubject differences in the susceptibility to MDE. G/NG contrasts were regressed against MDE magnitude (coded 1 for above and 0 for below the median MDE), with age, sex, and response time as covariates of no interest. The VS specifically showed higher G/NG modulation in subjects who exhibited greater MDE (Fig. 7A). VS activation was the only one to survive clusterwise FWE correction for multiple comparisons. The direct correlation across subjects between VS activation and behavioral MDE was statistically significant ( $p<0.05$, robust regression test). We therefore searched for the significant predictors of intersubject variability in VS response to the G/NG manipulation using a GLM that included all individual forward coefficients (for both input and connectivity) estimated in our best DCM (Fig. $7 B$ ). The only statistically significant $(p<0.05)$ predictor was the PMC-VS connectivity coefficient. A direct robust regression confirmed that PMC-VS connectivity was significantly correlated across subjects to VS activation $(p<0.01)$. Thus, the PM$\mathrm{C}-\mathrm{VS}$ link, which represents the passage from MNS to BVS, appeared crucial in shaping the BVS response that, in turn, accounted for individual susceptibility to MDE.

We finally checked the validity of our connectivity model using iterative multiple regressions, which allow including the be- 


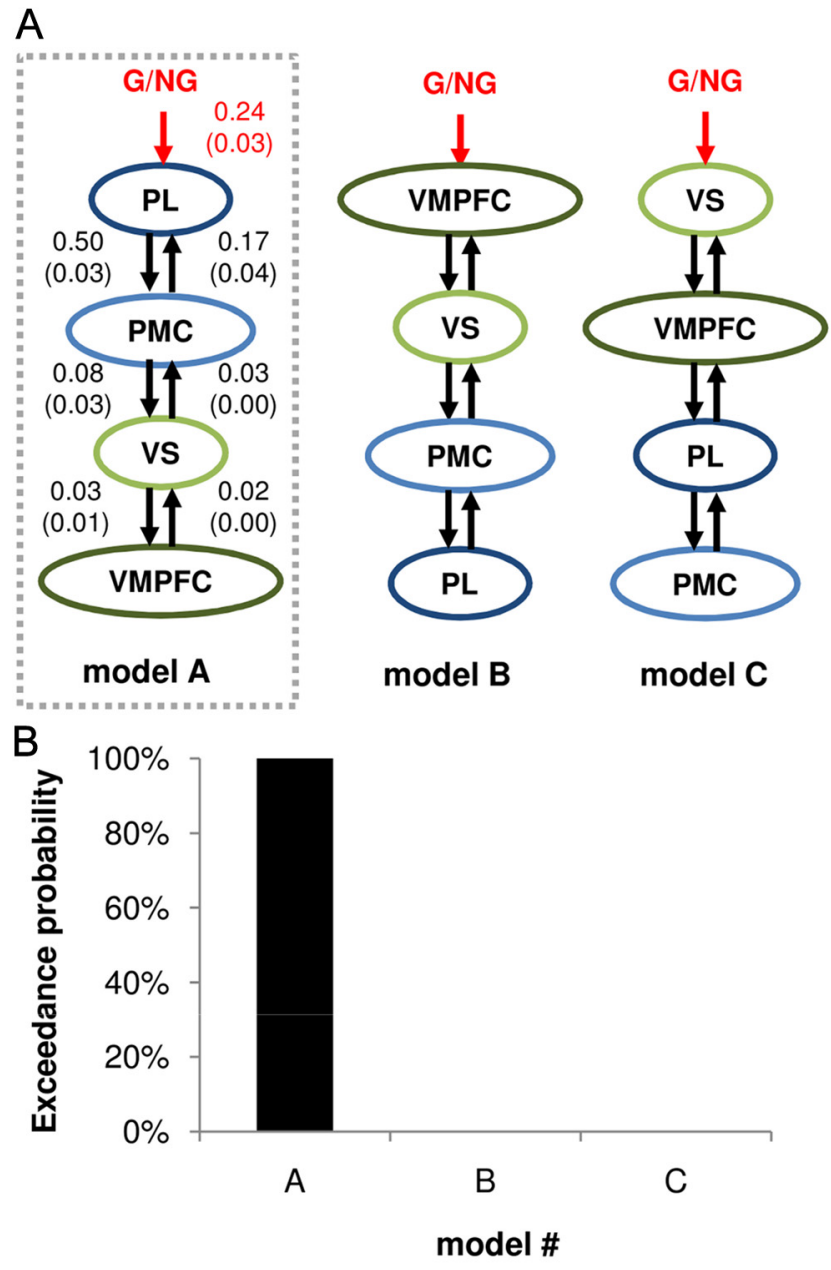

Figure 6. Direction of information transfer. Against the caudorostral linear model selected by previous model comparison (model $A$ ), we tested the reverse order (model $B$ ) and the inversion of the BVS (green) and MNS (blue) regions (model C). $\boldsymbol{A}$, Illustration of alternative DCMs. $\boldsymbol{B}$, Results of the BMS procedure. For the winning model (left), the mean \pm SD of posterior distributions obtained for the different modulation and connectivity coefficients are indicated. All coefficients were statistically significant at the group level. G/NG: video-viewing period modulated by the experimental condition ( 1 for goal, 0 for nongoal objects).

havioral response (desirability rating) in addition to experimental manipulation ( $\mathrm{G}$ vs NG) and brain activity. This analysis was performed on regression coefficients obtained from deconvolution of hemodynamic response to video display in our four regions of interest (PL, PMC, VS, and VMPFC). Between input (G vs NG video viewing) and output (desirability rating), the ROIs were serially ordered following a caudorostral progression, as in the best model identified by DCM analysis (Fig. 8). To test whether each of these regions contributed to the $\mathrm{MDE}$, we performed a series of multiple regressions that tested whether a given node explains a part of variance in the next node that is not explained by previous nodes (Table 4). The regression coefficient estimated for the last node was highly significant at each step of the iteration (all $p<0.001$, one-sample $t$ test), showing that all nodes of the model significantly contributed to the behavioral response. We do not claim, however, that the model is exhaustive; it is likely that other brain regions, such as visual and motor areas, also participate in the process that translates video viewing into desirability rating.

\section{Discussion}

We provide here empirical evidence for the MDE: observing a simple action directed toward an object increases the desir- ability of this object. The MDE appeared as a robust and general effect because it was repeatedly obtained in five groups of 24 participants each, regardless of age and sex, and for various kinds of objects (food, tools, clothes, and toys). It could have been expected that younger people have a higher susceptibility toward social influence. We observed this trend in our data, but it was not statistically significant. Given that we recruited only young adults (20-39 years), it remains possible that children and teenagers would exhibit even greater MDE. Debriefing suggested that the MDE can occur implicitly because most subjects remained unable to state their preference for objects presented as goals of actions. The seemingly implicit, automatic MDE found here contrasts with previous reports of goal contagion (Aarts et al., 2004), which used written description of goals that might trigger deliberate, strategic inferences.

An immediate question is in regard to MDE specificity. What exactly is more desirable: the pink candy that was grasped by the agent, any exemplar of an identical pink candy, or any sort of candy? Because it was obtained by a difference with respect to control objects that were identical except for colors, we conclude that the MDE can be very specific. However, the MDE was even greater when we considered only the first objects presented within each pair. This might come from a consistency bias, leading subjects to generalize their first rating to the second object of the pair and, hence, giving rise to the observed MDE reduction. It is also possible that color preferences were particularly strong in some subjects, resulting in a blurred MDE when calculated within pairs of differently colored objects. Note that color preferences cannot account for the group-level MDE because the colors assigned to $\mathrm{G}$ and NG objects were fully balanced across subjects. We discarded some potential confounds that might have driven the MDE, such as the occurrence of movement or the presence of a human agent in $\mathrm{G}$ videos, by comparing desirability ratings with appropriate control NG videos. We also addressed the possibility that $\mathrm{G}$ objects captured more attention using a recognition task, which confirmed that they were not better encoded in memory than NG objects.

We argue that the MDE is distinct from other phenomena that were documented previously. For instance, it is well known that humans tend to imitate the actions of others (Iacoboni, 2009). Here we focused our question on objects to assess whether watching goal-directed actions would increase the value of the object, not the propensity to imitate the action. Another trivial but pervasive phenomenon is that of suggestion, which is extensively exploited in commercials, either with verbal slogans or facial expressions, to increase products' desirability. Here there was no written information, and the agent's head was kept out of frame to avoid any suggestion effect. Leaving out the face also had the advantage of eliminating gaze processing and, hence, joint attention phenomena, which are known to influence judgment (Langton et al., 2000; Frischen et al., 2007). It has been shown that people tend to prefer objects that another agent looks at (Bayliss et al., 2006). This phenomenon may be closely related to the MDE investigated here because gazing could be viewed as analogous to reaching. However, an object is looked at when it attracts attention, possibly because it is desirable but also because it is salient, unusual, or even threatening. Reaching and grasping movements, therefore, convey more information about object values than eye movements. To our knowledge, the effects of gaze processing on preferences have not been investigated with functional neuroimaging. Further experiments are needed to examine whether these 

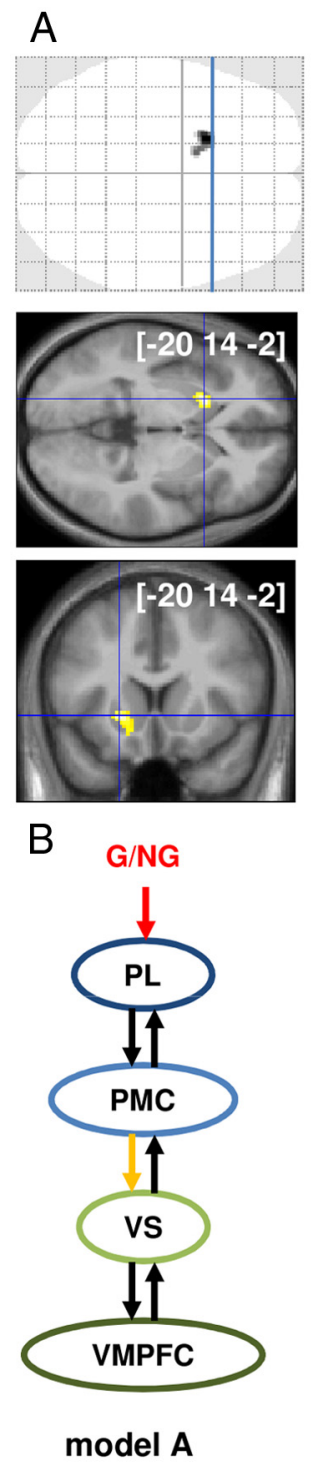
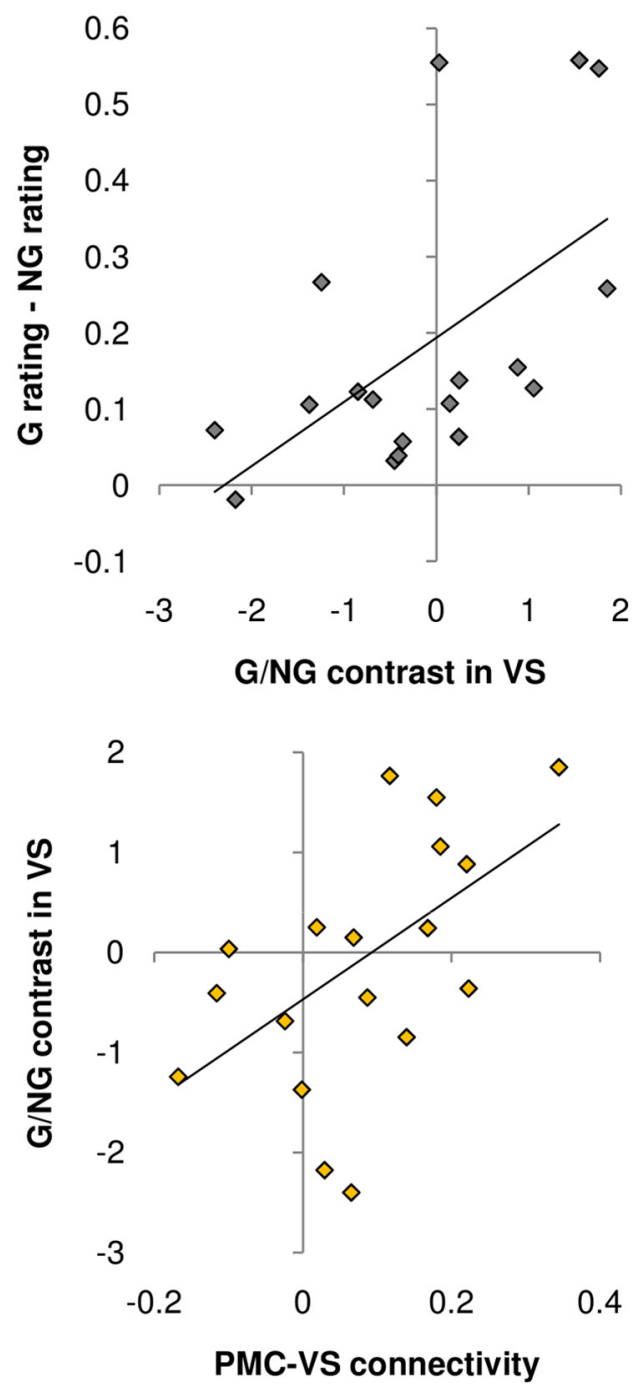

Figure 7. Interindividual differences. $\boldsymbol{A}$, Correlation between brain activation and MDE. Brain activation corresponds to the contrast between $\mathrm{G}$ and NG. MDE corresponds to the difference in Z-scored desirability ratings between $\mathrm{G}$ and NG objects. The activation map results from a group-level, between-subject regression of $\mathrm{G} / \mathrm{NG}$ contrast against behavioral MDE. Areas shown in gray/black on glass brains and in red/yellow on slices showed significant group-level random effects $(p<0.05$ after clusterwise FWE correction for multiple comparisons). The $[x, y, z]$ coordinates of the different maxima refer to the Montreal Neurological Institute space. Slices were taken at the maximum of interest, along planes indicated by blue lines on glass brains. The graph illustrates robust regression of behavioral MDE against G/NG contrast extracted from the maximum of interest in the VS. $\boldsymbol{B}$, Correlation between connectivity measures extracted from the best DCM and VS activation in the G/NG contrast. The graph illustrates robust regression of VS response to G/NG against the PMC-VS connectivity coefficient (yellow).

effects share common neural mechanisms with the MDE investigated here.

Our functional neuroimaging data suggest that the MDE involves an interaction between the MNS and the BVS, bridging two systems that have been characterized in separate bodies of literature as underpinning action observation and object valuation, respectively. Our results replicate classical findings that observing goal-directed actions activates the parietal lobules and premotor cortex (Iacoboni and Dapretto, 2006; Rizzolatti and Sinigaglia, 2010) and that subjective values correlate with the magnitude of ventral striatum and ventromedial prefrontal cortex activities (Rangel et al., 2008; Peters and Buchel, 2010). The novel finding is evidence that the influence of action observation on subjective values may involve MNS activity modulating BVS activity. This was supported by effective connectivity analyses using both intertrial and intersubject variance. DCM analyses validated the intuitive direction of information flow from visual inputs to motor outputs, that is, from the experimental manipulation (G vs NG video) to the behavioral effect (increased desirability rating). Crucially, forward connectivity from PMC to VS explained a significant part of intersubject differences in activating the BVS outputs while observing goaldirected actions. This is consistent with anatomical studies that showed, using axon tracing in monkeys and diffusion tensor imaging in humans, partial overlap between frontostriatal projections, such that motor frontal regions like the PMC can contact limbic striatal regions like the VS (Haber, 2003; Draganski et al., 2008).

In our opinion, these results do not speak to the current debate on the epistemic functions of the MNS. The original proposal was that MNS activation serves to understand an action "from the inside"; that is, to infer others' intentions by simulating their actions in our own motor system (Blakemore and Decety, 2001; Iacoboni and Dapretto, 2006; Rizzolatti and Sinigaglia, 2010). This view has been discussed, and alternative accounts have been suggested (Jacob and Jeannerod, 2005; Csibra and Gergely, 2007; Hickok, 2009). For instance, inferring others' intentions might rely on distinct regions that have been implicated in mentalizing, such as temporoparietal junction and medial prefrontal cortex (Frith and Frith, 2006; Saxe, 2006; Van Overwalle, 2009). Besides the alleged epistemic functions (knowing what others have in mind or what they will do next), our findings simply suggest that MNS activation is not neutral for the observer's own motivations; it will affect the values encoded in the BVS. The concept of mirror activations has already been extended from the motor to the emotional domain. Similar regions, such as the amygdala and insula, can be activated both when feeling an emotion and when observing someone manifesting an emotion (Carr et al., 2003; Keysers and Gazzola, 2006). These mechanisms might relate to the psychological concept of empathy (Decety and Jackson, 2004; Singer and Lamm, 2009). The phenomenon demonstrated here is different from emotional resonance because no emotion was expressed in the videos. The BVS regions that were modulated by the MNS in our data must be distinguished from the regions involved in emotional resonance (such as the insula and amygdala) and in social cognition (such as the temporoparietal junction or the medial prefrontal cortex, which is more dorsal than the VMPFC region discussed here). Accordingly, there was no correlation between the MDE and empathizing or mentalizing abilities, as measured by the EQ and the eyes test, respectively 


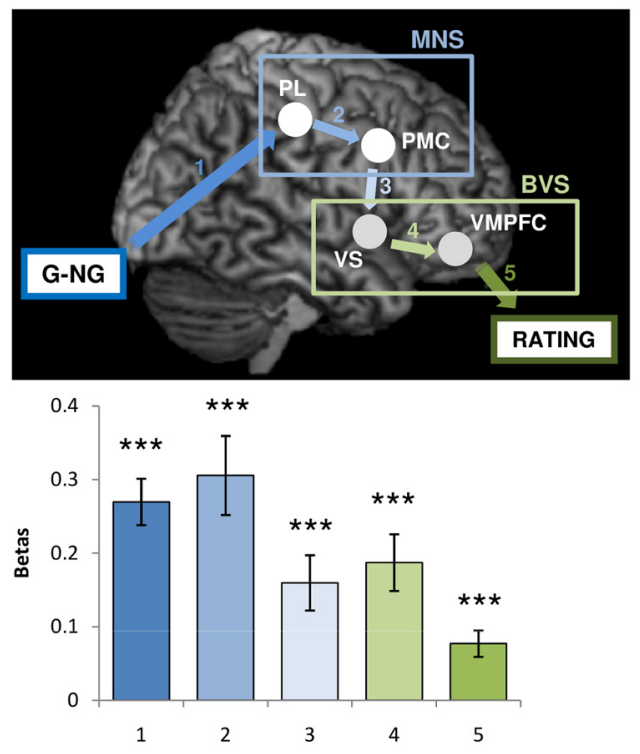

Figure 8. Functional connectivity model. Between the inputs ( $G$ or NG video) and outputs (desirability ratings), ROIs belonging to the mirror neuron system (PL and PMC) and to the brain valuation system (VS and VMPFC) were ordered along a caudorostral axis. Arrows indicate that a given node (experimental variable or brain activity) explains a significant part of the next node variance that is not explained by previous nodes. Histograms indicate the trial-by-trial regression coefficients obtained for the link between two successive nodes of the model, while controlling for the preceding nodes. Error bars indicate intersubject SEM. ${ }^{* * *} p<0.001$; one-sample $t$ test.

(Baron-Cohen, 2009). The central role of the VS, whose activation significantly accounted for individual susceptibility to the MDE, confirms that the brain system modulated here by the MNS underpins motivational functions. Indeed, VS activation has been consistently shown to represent expected rewards or subjective desirability, which can be seen as goal values (Haber and Knutson, 2010; Peters and Buchel, 2010). What we add here is a novel mechanism with which to form value representations in the VS and VMPFC through action observation.

We further suggest that MNS-BVS interaction constitutes an important mechanism serving to propagate values across individuals. This propagation could be incidental because the agents in the video had no intention to communicate desirability, nor did they truly experience a desire for the object. Given that it can be engaged in nonverbal situations, the MNS-BVS interaction might have been fundamental for many species, including our evolutionary ancestors, to share value information about objects present in the environment. Escaping the necessity of trial-anderror experience, including time and risk saving, is a potential advantage of this mechanism. A drawback could be that motivational contagion may lead to rivalry, which would make problematic the distribution of resources, and it may lead to herding, which would result in irrational mob behaviors (Girard, 1988; Raafat et al., 2009). The MNS-BVS interaction might constitute an essential mechanism through development, before children's language skills enable more sophisticated ways of expressing and suggesting values. A dysfunction in this mechanism would compromise the possibility of sharing desires and, hence, lead to social isolation. Many studies have shown that the MNS is dysfunctional in children with autistic spectrum disorders (Dapretto et al., 2006; Cattaneo et al., 2007), but some contradictory findings have also been reported (Hamilton et al., 2007; Dinstein et al., 2010). Another possibility is that the MNS may be functionally disconnected from the BVS in autistic patients, such that others' behavior would not affect their motivational system. This hypothesis would be in line with disconnection theories of autism and may call for further investigation.

\section{References}

Aarts H, Gollwitzer PM, Hassin RR (2004) Goal contagion: perceiving is for pursuing. J Pers Soc Psychol 87:23-37.

Baron-Cohen S (2009) Autism: the empathizing-systemizing (E-S) theory. Ann N Y Acad Sci 1156:68-80.

Bayliss AP, Paul MA, Cannon PR, Tipper SP (2006) Gaze cuing and affective judgments of objects: I like what you look at. Psychon Bull Rev 13:1061-1066.

Blakemore SJ, Decety J (2001) From the perception of action to the understanding of intention. Nat Rev Neurosci 2:561-567.

Carr L, Iacoboni M, Dubeau MC, Mazziotta JC, Lenzi GL (2003) Neural mechanisms of empathy in humans: a relay from neural systems for imitation to limbic areas. Proc Natl Acad Sci U S A 100:5497-5502.

Cattaneo L, Fabbri-Destro M, Boria S, Pieraccini C, Monti A, Cossu G, Rizzolatti G (2007) Impairment of actions chains in autism and its possible role in intention understanding. Proc Natl Acad Sci U S A 104:17825-17830.

Chib VS, Rangel A, Shimojo S, O’Doherty JP (2009) Evidence for a common representation of decision values for dissimilar goods in human ventromedial prefrontal cortex. J Neurosci 29:12315-12320.

Csibra G, Gergely G (2007) "Obsessed with goals": functions and mechanisms of teleological interpretation of actions in humans. Acta Psychol (Amst) 124:60-78.

Dapretto M, Davies MS, Pfeifer JH, Scott AA, Sigman M, Bookheimer SY, Iacoboni M (2006) Understanding emotions in others: mirror neuron dysfunction in children with autism spectrum disorders. Nat Neurosci 9:28-30.

Daunizeau J, David O, Stephan KE (2011) Dynamic causal modelling: a critical review of the biophysical and statistical foundations. Neuroimage 58:312-322.

Decety J, Jackson PL (2004) The functional architecture of human empathy. Behav Cogn Neurosci Rev 3:71-100.

Deichmann R, Gottfried JA, Hutton C, Turner R (2003) Optimized EPI for fMRI studies of the orbitofrontal cortex. Neuroimage 19:430-441.

Dinstein I, Thomas C, Humphreys K, Minshew N, Behrmann M, Heeger DJ (2010) Normal movement selectivity in autism. Neuron 66:461-469.

Draganski B, Kherif F, Klöppel S, Cook PA, Alexander DC, Parker GJ, Deichmann R, Ashburner J, Frackowiak RS (2008) Evidence for segregated and integrative connectivity patterns in the human basal ganglia. J Neurosci 28:7143-7152.

Frischen A, Bayliss AP, Tipper SP (2007) Gaze cueing of attention: visual attention, social cognition, and individual differences. Psychol Bull 133:694-724.

Frith CD, Frith U (2006) The neural basis of mentalizing. Neuron 50:531-534

Girard R (1988) Violence and the sacred. London: Athlone.

Gollwitzer PM, Moskowitz GB (1996) Goal effects on action and cognition. In: Social psychology: handbook of basic principles (Higgins ET, Kruglanski AW, eds), pp 361-399. New-York: Guilford.

Grèzes J, Armony JL, Rowe J, Passingham RE (2003) Activations related to 'mirror' and 'canonical' neurones in the human brain: an fMRI study. Neuroimage 18:928-937.

Haber SN (2003) The primate basal ganglia: parallel and integrative networks. J Chem Neuroanat 26:317-330.

Haber SN, Knutson B (2010) The reward circuit: linking primate anatomy and human imaging. Neuropsychopharmacology 35:4-26.

Hamilton AF, Brindley RM, Frith U (2007) Imitation and action understanding in autistic spectrum disorders: how valid is the hypothesis of a deficit in the mirror neuron system? Neuropsychologia 45:1859-1868.

Hare TA, Camerer CF, Rangel A (2009) Self-control in decision-making involves modulation of the vmPFC valuation system. Science 324:646-648.

Harrison LM, Penny W, Daunizeau J, Friston KJ (2008) Diffusion-based spatial priors for functional magnetic resonance images. Neuroimage 41:408-423. 
Table 4. Connectivity measures obtained from iterative multiple regression analysis

\begin{tabular}{llllll}
\hline & G/NG & PL & PMC & VS & VMPFC \\
\hline PL & $0.27 \pm 0.3(<0.001)$ & & & & \\
PMC & $0.09 \pm 0.03(<0.01)$ & $0.31 \pm 0.05(<0.001)$ & & & \\
VS & $0.00 \pm 0.03(0.48)$ & $0.08 \pm 0.03(<0.01)$ & $0.16 \pm 0.04(<0.001)$ & & \\
VMPFC & $-0.04 \pm 0.03(0.08)$ & $0.05 \pm 0.04(0.14)$ & $0.06 \pm 0.03(0.06)$ & $0.19 \pm 0.04(<0.001)$ & $0.08 \pm 0.02(<0.001)$ \\
Rating & $0.20 \pm 0.05(<0.001)$ & $0.00 \pm 0.03(0.45)$ & $-0.01 \pm 0.02(0.33)$ & $0.00 \pm 0.02(0.48)$ & \\
\hline
\end{tabular}

Values are mean \pm SEM ( $p$ value). The corresponding connectivity model is illustrated in Figure 8.

Hickok G (2009) Eight problems for the mirror neuron theory of action understanding in monkeys and humans. J Cogn Neurosci 21:1229-1243.

Iacoboni M (2009) Neurobiology of imitation. Curr Opin Neurobiol 19:661-665

Iacoboni M, Dapretto M (2006) The mirror neuron system and the consequences of its dysfunction. Nat Rev Neurosci 7:942-951.

Iacoboni M, Woods RP, Brass M, Bekkering H, Mazziotta JC, Rizzolatti G (1999) Cortical mechanisms of human imitation. Science 286:2526-2528.

Jacob P, Jeannerod M (2005) The motor theory of social cognition: a critique. Trends Cogn Sci 9:21-25.

Johnson-Frey SH, Maloof FR, Newman-Norlund R, Farrer C, Inati S, Grafton ST (2003) Actions or hand-object interactions? Human inferior frontal cortex and action observation. Neuron 39:1053-1058.

Keysers C, Gazzola V (2006) Towards a unifying neural theory of social cognition. Prog Brain Res 156:379-401.

Langton SR, Watt RJ, Bruce I I (2000) Do the eyes have it? Cues to the direction of social attention. Trends Cogn Sci 4:50-59.

Lebreton M, Jorge S, Michel V, Thirion B, Pessiglione M (2009) An automatic valuation system in the human brain: evidence from functional neuroimaging. Neuron 64:431-439.

Levy I, Lazzaro SC, Rutledge RB, Glimcher PW (2011) Choice from nonchoice: predicting consumer preferences from blood oxygenation level-dependent signals obtained during passive viewing. J Neurosci 31:118-125.

Peters J, Büchel C (2010) Neural representations of subjective reward value. Behav Brain Res 213:135-141.
Raafat RM, Chater N, Frith C (2009) Herding in humans. Trends Cogn Sci 13:420-428.

Rangel A, Camerer C, Montague PR (2008) A framework for studying the neurobiology of value-based decision making. Nat Rev Neurosci 9:545-556.

Rizzolatti G, Sinigaglia C (2010) The functional role of the parieto-frontal mirror circuit: interpretations and misinterpretations. Nat Rev Neurosci $11: 264-274$.

Saxe R (2006) Uniquely human social cognition. Curr Opin Neurobiol 16:235-239.

Singer T, Lamm C (2009) The social neuroscience of empathy. Ann N Y Acad Sci 1156:81-96.

Stephan KE, Penny WD, Daunizeau J, Moran RJ, Friston KJ (2009) Bayesian model selection for group studies. Neuroimage 46:1004-1017.

Van Overwalle F (2009) Social cognition and the brain: a meta-analysis. Hum Brain Mapp 30:829-858.

Van Overwalle F, Baetens K (2009) Understanding others' actions and goals by mirror and mentalizing systems: a meta-analysis. Neuroimage 48:564-584.

Von Neumann J, Morgenstern O (1944) Theory of games and economic behavior. Princeton: Princeton UP.

Weiskopf N, Hutton C, Josephs O, Deichmann R (2006) Optimal EPI parameters for reduction of susceptibility-induced BOLD sensitivity losses: a whole-brain analysis at $3 \mathrm{~T}$ and $1.5 \mathrm{~T}$. Neuroimage 33:493504 . 